\title{
Parabolic potentials and wavelet transforms with the generalized translation
}

\author{
by \\ Ilham A. Aliev (Antalya) and Boris Rubin (Jerusalem)
}

\begin{abstract}
Parabolic wavelet transforms associated with the singular heat operators $-\Delta_{\gamma}+\partial / \partial t$ and $I-\Delta_{\gamma}+\partial / \partial t$, where $\Delta_{\gamma}=\sum_{k=1}^{n} \partial^{2} / \partial x_{k}^{2}+\left(2 \gamma / x_{n}\right) \partial / \partial x_{n}$, are introduced. These transforms are defined in terms of the relevant generalized translation operator. An analogue of the Calderón reproducing formula is established. New inversion formulas are obtained for generalized parabolic potentials representing negative powers of the singular heat operators.
\end{abstract}

1. Introduction. The continuous wavelet transforms

$$
f(x) \mapsto W f(x, t)=\frac{1}{t^{n}} \int_{\mathbb{R}^{n}} f(y) w\left(\frac{|x-y|}{t}\right) d y,
$$

where $x \in \mathbb{R}^{n}, t \in(0, \infty), \int_{\mathbb{R}^{n}} w(|x|) d x=0$, and the corresponding Calderóntype reproducing formula

$$
\int_{0}^{\infty} W f(x, t) \frac{d t}{t}=c_{w} f(x),
$$

were studied by many authors in connection with various applications (see, e.g., [14] for further references). The more general formula

$$
\int_{0}^{\infty} W f(x, t) \frac{d t}{t^{1+\alpha}}=c_{\alpha, w}(-\Delta)^{\alpha / 2} f(x), \quad \alpha \in \mathbb{C},
$$

gives integral representations of complex powers of $-\Delta$ where $\Delta$ is the Laplacian in $\mathbb{R}^{n}$. In the present article we extend these results to the case when

2000 Mathematics Subject Classification: Primary 42C40.

Key words and phrases: parabolic wavelet transforms, parabolic potentials, generalized translation operator, singular heat operators, Calderón's reproducing formula.

The second coauthor was partially supported by the Edmund Landau Center for Research in Mathematical Analysis and Related Areas, sponsored by the Minerva Foundation (Germany). 
$\Delta$ is replaced by the singular heat operators

$$
-\Delta_{\gamma}+\frac{\partial}{\partial t} \quad \text { and } \quad I-\Delta_{\gamma}+\frac{\partial}{\partial t}, \quad \Delta_{\gamma}=\sum_{k=1}^{n} \frac{\partial^{2}}{\partial x_{k}^{2}}+\frac{2 \gamma}{x_{n}} \frac{\partial}{\partial x_{n}} .
$$

We introduce new wavelet transforms which reflect anisotropy and the character of the singularity in (1.4) simultaneously. The problem was inspired, on the one hand, by the earlier papers of A. Weinstein, M. N. Olevskil and other authors (see, e.g., [24, 25]) related to singular PDE and the corresponding generalized axially symmetric problems. On the other hand, our study was motivated by needs of Fourier-Bessel harmonic analysis which employs the notion of the generalized translation operator and goes back to S. Bochner, J. Delsarte, B. M. Levitan, and others (see [8-11, 20, 21, 23] for further details and references). Discrete wavelet systems were studied by many authors; see, e.g., the nice book of P. Wojtaszczyk [26] and references therein.

The plan of the paper is as follows. Some necessary definitions and auxiliary facts are given in Section 2. In particular, we introduce new notions of "generalized parabolic wavelet transforms" associated with the singular heat operators (1.4). In Section 3 we obtain inversion formulas (Calderóntype reproducing formulas) for these transforms. The generalized parabolic potentials associated with (1.4) and defined in Fourier-Bessel terms by

$$
\begin{aligned}
& \left(H_{\gamma}^{\alpha} f\right)^{\wedge}(x, t)=\left(|x|^{2}+i t\right)^{-\alpha / 2} f^{\wedge}(x, t), \\
& \left(\mathcal{H}_{\gamma}^{\alpha} f\right)^{\wedge}(x, t)=\left(1+|x|^{2}+i t\right)^{-\alpha / 2} f^{\wedge}(x, t),
\end{aligned}
$$

are studied in Section 4. We obtain wavelet-type representations of these potentials, like (1.3), and prove the relevant $\left(L^{p}-L^{q}\right)$-estimates of the HardyLittlewood-Sobolev type. Section 5 is devoted to the inversion of the generalized parabolic potentials by means of the relevant continuous wavelet transforms introduced in Section 2.

Historical remarks. Parabolic potentials associated with ordinary (nonsingular) heat operators were introdiced by B. F. Jones and C. H. Sampson $[7,17]$. Various properties of these potentials and the relevant anisotropic function spaces of the Sobolev type were studied by R. Bagby, V. R. Gopala Rao, S. Chanillo, V. A. Nogin and B. S. Rubin (see $[12,16]$ and references therein). Continuous wavelet transforms corresponding to these potentials were introduced in the authors' paper [4]. Singular parabolic equations were studied by many authors (see, e.g., [5, 22]). The relevant parabolic potentials were introduced by I. Aliev [1,2]. The wavelet approach to these potentials, developed below, seems to be new. In subsequent publications we plan to give some applications of our results to singular parabolic equations. 
2. Preliminaries. Let $x=\left(x_{1}, \ldots, x_{n-1}, x_{n}\right) \equiv\left(x^{\prime}, x_{n}\right) \in \mathbb{R}^{N} ; \mathbb{R}_{+}^{n}=$ $\left\{x \in \mathbb{R}^{n}: x_{n}>0\right\} ; \dot{\mathbb{R}}_{+}^{n}=\left\{x \in \mathbb{R}^{n}: x_{n} \geq 0\right\} ; d \nu(x)=x_{n}^{2 \gamma} d x, \gamma>0$. The Fourier-Bessel transform of a sufficiently nice function $f: \mathbb{R}_{+}^{n} \rightarrow \mathbb{C}$ and its inverse are defined by

$$
\begin{gathered}
f^{\wedge}(x) \equiv\left(F_{\gamma} f\right)(x)=\int_{\mathbb{R}_{+}^{n}} f(y) e^{-i x^{\prime} \cdot y^{\prime}} j_{\gamma-1 / 2}\left(x_{n} y_{n}\right) d \nu(y), \\
F_{\gamma}^{-1} f(x)=c_{\gamma}^{2}(n)\left(F_{\gamma} f\right)\left(-x^{\prime}, x_{n}\right), \quad x=\left(x^{\prime}, x_{n}\right) \in \mathbb{R}_{+}^{n}, \\
c_{\gamma}(n)=\left[2^{\gamma-1 / 2}(2 \pi)^{(n-1) / 2} \Gamma(\gamma+1 / 2)\right]^{-1},
\end{gathered}
$$

where $j_{\lambda}(z)=2^{\lambda} \Gamma(\lambda+1) z^{-\lambda} J_{\lambda}(z), \lambda>-1 / 2$, is the normalized Bessel function such that $j_{\lambda}(0)=1$ (see, e.g., $[8,9]$ ). The same definition was applied in (1.5) and (1.6) to functions on $\mathbb{R}_{+}^{n} \times \mathbb{R}^{1}=\left\{\left(x_{1}, \ldots, x_{n}, t\right) \in\right.$ $\left.\mathbb{R}^{n+1}: x_{n}>0\right\}$.

We need the following weighted $L^{p}$-spaces, $1 \leq p<\infty$ :

$$
\begin{aligned}
& X_{p} \equiv L^{p}\left(\mathbb{R}_{+}^{n} ; d \nu(x)\right)=\left\{g:\|g\|_{X_{p, \gamma}}=\left(\int_{\mathbb{R}_{+}^{n}}|g(x)|^{p} d \nu(x)\right)^{1 / p}<\infty\right\}, \\
& Y_{p} \equiv L^{p}\left(\mathbb{R}_{+}^{n} \times \mathbb{R}^{1} ; d \nu(x) d t\right)=\left\{f:\left(\int_{\mathbb{R}_{+}^{n} \times \mathbb{R}^{1}}|f(x, t)|^{p} d \nu(x) d t\right)^{1 / p}<\infty\right\} .
\end{aligned}
$$

In the case $p=\infty$ we identify $L^{\infty}$ with $C^{0}$, the corresponding space of continuous functions vanishing at infinity.

Given a finite (signed) Borel measure $m \equiv m(y, \tau), y \in \dot{\mathbb{R}}_{+}^{n}, \tau \in \mathbb{R}^{1}$, and $\eta>0$, we define an anisotropic dilation $m_{\sqrt{\eta}, \eta}$ of $m$ by

$$
\int_{\dot{R}_{+}^{n} \times \mathbb{R}^{1}} \omega(y, \tau) d m_{\sqrt{\eta}, \eta}(y, \tau)=\int_{\mathbb{R}_{+}^{n} \times \mathbb{R}^{1}} \omega(\sqrt{\eta} y, \eta \tau) d m(y, \tau)
$$

for $\omega \in C^{0} \equiv C^{0}\left(\dot{\mathbb{R}}_{+}^{n} \times \mathbb{R}^{1}\right)$. In particular, for $d m(y, \tau)=\varphi(y, \tau) d \nu(y) d \tau$ with $\varphi \in Y_{1}$, we have

$$
\begin{aligned}
d m_{\sqrt{\eta}, \eta}(y, \tau) & =\varphi_{\sqrt{\eta}, \eta}(y, \tau) d \nu(y) d \tau \\
\varphi_{\sqrt{\eta}, \eta}(y, \tau) & =(\sqrt{\eta})^{-(n+2 \gamma+2)} \varphi\left(\frac{y}{\sqrt{\eta}}, \frac{\tau}{\eta}\right) .
\end{aligned}
$$

For $x \in \mathbb{R}_{+}^{n}, y \in \dot{\mathbb{R}}_{+}^{n}$, and $t, \tau \in \mathbb{R}^{1}$, the generalized translation of $f$ : $\mathbb{R}_{+}^{n} \times \mathbb{R}^{1} \rightarrow \mathbb{C}$ is defined by

$$
\begin{array}{r}
T^{y, \tau} f(x, t)=\frac{\Gamma(\gamma+1 / 2)}{\Gamma(\gamma) \Gamma(1 / 2)} \int_{0}^{\pi} f\left(x^{\prime}-y^{\prime} ; \sqrt{x_{n}^{2}-2 x_{n} y_{n} \cos \beta+y_{n}^{2}} ; t-\tau\right) \\
\times \sin ^{2 \gamma-1} \beta d \beta
\end{array}
$$


(cf. $[8,9,23])$. Here we actually deal with the ordinary translation in $x^{\prime}$ and $t$, and with the generalized translation in $x_{n}$. For the relevant one-dimensional operator

$$
S^{\varrho} g(r)=\frac{\Gamma(\gamma+1 / 2)}{\Gamma(\gamma) \Gamma(1 / 2)} \int_{0}^{\pi} g\left(\sqrt{r^{2}-2 r \varrho \cos \beta+\varrho^{2}}\right) \sin ^{2 \gamma-1} \beta d \beta
$$

the following relations are known [9]:

$$
\begin{gathered}
S^{\varrho} g(r)=S^{r} g(\varrho), \quad S^{\varrho} S^{\tau} g(r)=S^{\tau} S^{\varrho} g(r), \\
S^{\varrho} g(r)=S^{-\varrho} g(r), \quad S^{0} g(r)=g(r), \\
\int_{0}^{\infty} f(r) S^{r} g(\varrho) r^{2 \gamma} d r=\int_{0}^{\infty} S^{r} f(\varrho) g(r) r^{2 \gamma} d r .
\end{gathered}
$$

Note also that for $1 \leq p \leq \infty$,

$$
\begin{gathered}
\left\|T^{y, \tau} f\right\|_{Y_{p}} \leq\|f\|_{Y_{p}}, \\
\left\|T^{y, \tau} f-f\right\|_{Y_{p}} \rightarrow 0 \quad \text { as }(y, \tau) \rightarrow(0,0) \in \dot{\mathbb{R}}_{+}^{n} \times \mathbb{R}^{1},
\end{gathered}
$$

$Y_{\infty}$ being identified with $C^{0}$ (see, e.g., [10]).

The generalized convolution associated with the generalized translation operator (2.4) is defined by

$$
(f \otimes g)(x, t)=\int_{\mathbb{R}_{+}^{n} \times \mathbb{R}^{1}} g(y, \tau) T^{y, \tau} f(x, t) d \nu(y) d \tau, \quad(x, t) \in \mathbb{R}_{+}^{n} \times \mathbb{R}^{1} .
$$

By using (2.8) and the Riesz-Thorin interpolation theorem it is not difficult to prove the corresponding Young inequality

$$
\|f \otimes g\|_{Y_{r}} \leq\|f\|_{Y_{p}}\|g\|_{Y_{q}}, \quad 1 \leq p, q, r \leq \infty, \frac{1}{p}+\frac{1}{q}=\frac{1}{r}+1 .
$$

Similarly to $(2.10)$, for a finite Borel measure $m=m(y, \tau)$ on $\dot{\mathbb{R}}_{+}^{n} \times \mathbb{R}^{1}$ we define

$$
(f \otimes m)(x, t)=\int_{\mathbb{R}_{+}^{n} \times \mathbb{R}^{1}} T^{y, \tau} f(x, t) d m(y, \tau), \quad(x, t) \in \mathbb{R}_{+}^{n} \times \mathbb{R}^{1} .
$$

Definition 2.1. Given a finite (signed) Borel measure $m$ such that $m\left(\dot{\mathbb{R}}_{+}^{n} \times \mathbb{R}^{1}\right)=0$, a generalized anisotropic continuous wavelet transform of $f: \mathbb{R}_{+}^{n} \times \mathbb{R}^{1} \rightarrow \mathbb{C}$ is defined by

$$
\begin{aligned}
& V_{m} f(x, t ; \eta)=\left(f \otimes m_{\sqrt{\eta}, \eta}\right)(x, t) \equiv \int_{\mathbb{R}_{+}^{n} \times \mathbb{R}^{1}} T^{\sqrt{\eta} y, \eta \tau} f(x, t) d m(y, \tau), \\
& (x, t) \in \mathbb{R}_{+}^{n} \times \mathbb{R}^{1}, \eta>0 .
\end{aligned}
$$


The choice of the "wavelet measure" $m$ is at our disposal. We specify $m$ as follows. Let us introduce the generalized Gauss-Weierstrass kernel

$$
W_{\gamma}(y, s)=c_{\gamma}(n)(2 s)^{-(n+2 \gamma) / 2} \exp \left(-|y|^{2} /(4 s)\right), \quad y \in \mathbb{R}_{+}^{n}, s>0,
$$

$c_{\gamma}(n)$ being defined by (2.2) (see [20] for $n=1$ and [2] for any $n \geq 1$ ).

Lemma 2.2. (a) $\left(F_{\gamma} W_{\gamma}(\cdot, s)\right)(x)=\exp \left(-s|x|^{2}\right)$.

(b) $W_{\gamma}(y, s)$ is an anisotropic $(1 / 2,1)$-homogeneous function of order $k=-\gamma-n / 2$, i.e.

$$
W_{\gamma}\left(\lambda^{1 / 2} y, \lambda s\right)=\lambda^{-\gamma-n / 2} W_{\gamma}(y, s), \quad \forall y \in \mathbb{R}_{+}^{n}, s>0, \lambda>0 .
$$

In particular,

$$
W_{\gamma}\left(\lambda^{1 / 2} y, \lambda\right)=\lambda^{-\gamma-n / 2} W_{\gamma}(y, 1)
$$

(c) We have

$$
\left\|W_{\gamma}(\cdot, s)\right\|_{X_{1}} \equiv \int_{\mathbb{R}_{+}^{n}} W_{\gamma}(y, s) d \nu(y)=1 .
$$

(d) Moreover,

$$
\begin{aligned}
& \left(W_{\gamma}(\cdot, \tau) \otimes W_{\gamma}(\cdot, t)\right)(x) \\
& \quad \equiv \int_{\mathbb{R}_{+}^{n}} W_{\gamma}(y, \tau) T^{y} W_{\gamma}(x, t) d \nu(y)=W_{\gamma}(x, t+\tau),
\end{aligned}
$$

$T^{y}$ being the generalized translation operator in the $x$-variable (cf. (2.4)).

Proof. (a) is a consequence of the formulas

$$
\begin{gathered}
\int_{\mathbb{R}^{n}} e^{-s|x|^{2}} e^{-i x \cdot y} d x=(\pi / s)^{n / 2} \exp \left(-|y|^{2} /(4 s)\right), \\
\int_{0}^{\infty} e^{-s r^{2}} r^{\gamma+1} J_{\gamma}(t r) d r=t^{\gamma}(2 s)^{-\gamma-1} \exp \left(-t^{2} /(4 s)\right) ;
\end{gathered}
$$

(b) is obvious; (c) follows from (a); (d) is clear in terms of the Fourier-Bessel transform because $F_{\gamma}(\varphi \otimes \psi)=\left(F_{\gamma} \varphi\right)\left(F_{\gamma} \psi\right)$.

Given a function $g: \mathbb{R}_{+}^{n} \rightarrow \mathbb{C}$, we introduce the generalized GaussWeierstrass integral

$$
\left(G_{\gamma} g\right)(x, s)=\left(g \otimes W_{\gamma}(\cdot, s)\right)(x)=\int_{\mathbb{R}_{+}^{n}} T^{y} g(x) W_{\gamma}(y, s) d \nu(y),
$$

and the $\gamma$-maximal function

$$
M_{\gamma} g(x)=\sup _{r>0} \frac{1}{r^{n+2 \gamma} \omega(n, \gamma)} \int_{B_{r}^{+}}\left|T^{x} g(y)\right| d \nu(y),
$$


where

$$
B_{r}^{+}=\left\{y: y \in \mathbb{R}_{+}^{n},|y| \leq r\right\}, \quad \omega(n, \gamma)=\int_{B_{1}^{+}} d \nu(y)
$$

so that

$$
\begin{gathered}
\left\|M_{\gamma} g\right\|_{X_{\varrho}} \leq c\|g\|_{X_{\varrho}}, \quad 1<p \leq \infty, \\
\sup _{s>0}\left|G_{\gamma} g(x, s)\right| \leq M_{\gamma} g(x)
\end{gathered}
$$

(see [20, 21] and [3, p. 370]).

Now we specify $m$ in Definition 2.1. Let $\mu$ be a finite Borel measure on $\mathbb{R}^{1}$ such that $\mu\left(\mathbb{R}^{1}\right)=0$ and $\operatorname{supp} \mu \subset[0, \infty)$. Define $\mu^{\prime}=\mu-\mu(\{0\}) \delta$, where $\delta=\delta(\tau)$ is the unit mass at $\tau=0$, and set

$$
m=\mu(\{0\}) \delta(y, \tau)+m^{\prime}, \quad d m^{\prime}(y, \tau)=W_{\gamma}(y, \tau) d \nu(y) d \mu^{\prime}(\tau),
$$

where $\delta(y, \tau)$ is the unit mass at $(0,0) \in \dot{\mathbb{R}}_{+}^{n} \times \mathbb{R}^{1}$ and $W_{\gamma}(y, \tau)$ is extended by zero to $\tau<0$. Clearly, $m$ is finite and by (2.17),

$$
m\left(\dot{\mathbb{R}}_{+}^{n} \times \mathbb{R}^{1}\right)=\int_{\mathbb{R}^{1}} d \mu(\tau) \int_{\mathbb{R}_{+}^{n}} W_{\gamma}(y, \tau) d \nu(y)=\int_{\mathbb{R}^{1}} d \mu(\tau)=0 .
$$

Definition 2.3. The anisotropic wavelet transform (2.13) with $m$ defined by (2.23) will be called a generalized parabolic wavelet transform and denoted by $V_{\mu} f(x, t ; \eta)$. Thus

$$
V_{\mu} f(x, t ; \eta)=\int_{\mathbb{R}_{+}^{n} \times[0, \infty)} T^{\sqrt{\eta} y, \eta \tau} f(x, t) W_{\gamma}(y, \tau) d \nu(y) d \mu(\tau)
$$

or, by (2.16) and (2.17),

$$
\begin{aligned}
V_{\mu} f(x, t ; \eta)= & \int_{\mathbb{R}_{+}^{n} \times[0, \infty)} T^{\sqrt{\eta \tau} y, \eta \tau} f(x, t) W_{\gamma}(y, 1) d \nu(y) d \mu(\tau) \\
= & \mu(\{0\}) f(x, t) \\
& +\int_{\mathbb{R}_{+}^{n} \times(0, \infty)} T^{\sqrt{\eta \tau} y, \eta \tau} f(x, t) W_{\gamma}(y, 1) d \nu(y) d \mu(\tau) .
\end{aligned}
$$

Definition 2.4. The transform

$$
\begin{array}{r}
\mathcal{V}_{\mu} f(x, t ; \eta)=\int_{\mathbb{R}_{+}^{n} \times[0, \infty)} T^{\sqrt{\eta} y, \eta \tau} f(x, t) W_{\gamma}(y, \tau) e^{-\eta \tau} d \nu(y) d \mu(\tau) \\
\stackrel{(2.16)}{=} \int_{\mathbb{R}_{+}^{n} \times[0, \infty)} T^{\sqrt{\eta \tau} y, \eta \tau} f(x, t) W_{\gamma}(y, 1) e^{-\eta \tau} d \nu(y) d \mu(\tau)
\end{array}
$$

will be called a weighted generalized parabolic wavelet transform of $f$.

In the following we use the convention $\int_{a}^{b} \varphi(t) d \mu(t)=\int_{[a, b)} \varphi(t) d \mu(t)$. 


\section{Inversion of generalized parabolic wavelet transforms (the Calderón-type reproducing formula)}

Theorem 3.1. Let $V_{\mu} f(x, t ; \eta)$ be the wavelet transform (2.24), $f \in Y_{p}$, $1<p \leq \infty$ (recall that $Y_{\infty}$ is identified with $C^{0}$ ). Suppose that

$$
\begin{gathered}
\mu([0, \infty))=0, \quad \int_{0}^{\infty}|\log \tau| d|\mu|(\tau)<\infty, \\
c_{\mu}=\int_{0}^{\infty} \log \frac{1}{\tau} d \mu(\tau) .
\end{gathered}
$$

Then

$$
\int_{0}^{\infty} V_{\mu} f(x, t ; \eta) \frac{d \eta}{\eta} \equiv \lim _{\varepsilon \rightarrow 0, \varrho \rightarrow \infty} \int_{\varepsilon}^{\varrho} V_{\mu} f(x, t ; \eta) \frac{d \eta}{\eta}=c_{\mu} f(x, t),
$$

the limit being understood in the $Y_{p}$-norm. If moreover $1<p<\infty$ and

$$
\int_{0}^{1}|\log \tau|^{\delta} d|\mu|(\tau)<\infty \quad \text { for some } \delta>1,
$$

then the limit in (3.3) can be interpreted pointwise for almost all $(x, t) \in$ $\mathbb{R}_{+}^{n} \times \mathbb{R}^{1}$. The same statements hold for the weighted transform (2.26).

Proof. Define

$$
I_{\varepsilon, \varrho}(\mu, f)=\int_{\varepsilon}^{\varrho} V_{\mu} f(x, t ; \eta) \frac{d \eta}{\eta}, \quad 0<\varepsilon<\varrho<\infty .
$$

We have

$$
\begin{gathered}
I_{\varepsilon, \varrho}(\mu, f)=f \otimes K_{\varepsilon}-f \otimes K_{\varrho}, \\
K_{\varepsilon} \equiv K_{\varepsilon}(y, \tau)=W_{\gamma}(y, \tau) k_{\varepsilon}(\tau), \\
k_{\varepsilon}(\tau)=\frac{1}{\varepsilon} k\left(\frac{\tau}{\varepsilon}\right), \quad k(\tau)=\frac{1}{\tau} \int_{0}^{\tau} d \mu(s)=\frac{1}{\tau} \mu([0, \tau))
\end{gathered}
$$

(and similarly for $\left.K_{\varrho}\right)$. Indeed, by $(3.1), \mu(\{0\})=0$ so that

$$
\begin{aligned}
& I_{\varepsilon, \varrho}(\mu, f)(x, t) \stackrel{(2.24)}{=} \int_{0}^{\infty} d \mu(\tau) \int_{\varepsilon}^{\varrho} \frac{d \eta}{\eta} \int_{\mathbb{R}_{+}^{n}} T^{\sqrt{\eta} y, \eta \tau} f(x, t) W_{\gamma}(y, \tau) d \nu(y) \\
& \stackrel{(2.15)}{=} \int_{0}^{\infty} d \mu(\tau) \int_{\varepsilon \tau}^{\varrho \tau} \frac{d s}{s} \int_{\mathbb{R}_{+}^{n}} T^{z, s} f(x, t) W_{\gamma}(z, s) d \nu(z)
\end{aligned}
$$




$$
\begin{aligned}
& =\int_{0}^{\infty} \frac{d s}{s} \int_{s / \varrho}^{s / \varepsilon} d \mu(\tau) \int_{\mathbb{R}_{+}^{n}} T^{z, s} f(x, t) W_{\gamma}(z, s) d \nu(z) \\
& =\int_{\mathbb{R}_{+}^{n} \times(0, \infty)} T^{z, s} f(x, t) W_{\gamma}(z, s)\left[\frac{1}{s} \int_{s / \varrho}^{s / \varepsilon} d \mu(\tau)\right] d \nu(z) d(s),
\end{aligned}
$$

which gives (3.6).

Let $\varepsilon \rightarrow 0$ and $\varrho \rightarrow \infty$ in (3.6). By $(2.15)$,

$$
\left(f \otimes K_{\varepsilon}\right)(x, t)=\int_{\mathbb{R}_{+}^{n} \times(0, \infty)} T^{\sqrt{\varepsilon} y, \varepsilon \tau} f(x, t) W_{\gamma}(y, \tau) k(\tau) d \nu(y) d \tau .
$$

It is known that $(3.1)$ implies $k \in L_{1}(0, \infty)$ and $\int_{0}^{\infty} k(\tau) d \tau=c_{\mu}$ (see [13], p. 188). Hence, by (2.17),

$$
\left\|f \otimes K_{\varepsilon}-c_{\mu} f\right\|_{Y_{p}} \leq \int_{\mathbb{R}_{+}^{n} \times(0, \infty)} W_{\gamma}(y, \tau)|k(\tau)| \cdot\left\|T^{\sqrt{\varepsilon} y, \varepsilon \tau} f-f\right\|_{Y_{p}} d \nu(y) d \tau .
$$

Owing to (2.19), (2.9) and the Lebesgue dominated convergence theorem, this yields $\lim _{\varepsilon \rightarrow 0}\left\|f \otimes K_{\varepsilon}-c_{\mu} f\right\|_{Y_{p}}=0,1 \leq p \leq \infty$. To complete the proof of the first statement we note that $\lim _{\varrho \rightarrow \infty}\left\|f \otimes K_{\varrho}\right\|_{Y_{p}}=0$. Indeed, by (2.8) and (2.17), we have

$$
\left\|f \otimes K_{\varrho}\right\|_{Y_{p}} \leq\left\|\int_{0}^{\infty}|k(\tau)| g(t-\varrho \tau) d \tau\right\|_{L^{p}\left(\mathbb{R}_{t}^{1}\right)}
$$

where $g(t)=\|f(\cdot, t)\|_{X_{p}} \in L^{p}\left(\mathbb{R}^{1}\right)$, and the result follows in a standard way (see, e.g., Theorem 1.15 of [13]).

Let us prove the a.e. convergence in (3.3). By (2.19),

$$
\begin{aligned}
\left|\left(f \otimes K_{\varepsilon}\right)(x, t)\right| & \leq \int_{0}^{\infty}|k(\tau)| d \tau \int_{\mathbb{R}_{+}^{n}} W_{\gamma}(y, \varepsilon \tau) T_{x}^{y}|f|(x, t-\varepsilon \tau) d \nu(y) \\
& =\int_{0}^{\infty}|k(\tau)| G_{\gamma}(|f(\cdot, t-\varepsilon \tau)|)(x, \varepsilon \tau) d \tau \\
& \leq \int_{0}^{\infty}|k(\tau)| \sup _{s>0} G_{\gamma}(|f(\cdot, t-\varepsilon \tau)|)(x, s) d \tau \\
& \leq \int_{0}^{\infty}|k(\tau)| M_{\gamma}[|f(\cdot, t-\varepsilon \tau)|](x) d \tau
\end{aligned}
$$

By (3.1) and (3.4), the function $k(t)=t^{-1} \int_{0}^{t} d \mu(\tau) \equiv-t^{-1} \int_{t}^{\infty} d \mu(\tau)$ has a 
decreasing integrable majorant, and therefore

$$
F(x, t):=\sup _{\varepsilon>0}\left|\left(f \otimes K_{\varepsilon}\right)(x, t)\right| \leq c\left(M u_{x}\right)(t),
$$

where $u_{x}(t)=M_{\gamma}[|f(\cdot, t)|](x)$, with the standard one-dimensional HardyLittlewood maximal function $M$ in the $t$-variable [19, p. 59]. Hence

$$
\begin{aligned}
\|F(x, t)\|_{Y_{p}} & =\|\| F(x, \cdot)\left\|_{L^{p}\left(\mathbb{R}^{1}\right)}\right\|_{X_{p}} \stackrel{(3.10)}{\leq} c\|\| u_{x}(\cdot)\left\|_{L^{p}\left(\mathbb{R}^{1}\right)}\right\|_{X_{p}} \\
& =c\|\| u_{x}(t)\left\|_{X_{p}}\right\|_{L^{p}\left(\mathbb{R}^{1}\right)} \stackrel{(2.21)}{\leq} c\|\| f(\cdot, t)\left\|_{X_{p}}\right\|_{L^{p}\left(\mathbb{R}^{1}\right)}=c\|f\|_{Y_{p}}
\end{aligned}
$$

(here and below the constant $c$ may be different at each occurrence). Thus

$$
\left\|\sup _{\varepsilon>0}\left|f \otimes K_{\varepsilon}\right|\right\|_{Y_{p}} \leq c\|f\|_{Y_{p}}, \quad 1<p \leq \infty .
$$

Owing to this estimate and taking into account that

$$
\left(f \otimes K_{\varepsilon}\right)(x, t) \equiv \int_{\mathbb{R}_{+}^{n} \times(0, \infty)} W_{\gamma}(y, \tau) k(\tau) T^{\sqrt{\varepsilon} y, \varepsilon \tau} f(x, t) d \nu(y) d \tau \rightarrow f(x, t)
$$

pointwise as $\varepsilon \rightarrow 0$ for any $f \in C^{0}\left(\mathbb{R}_{+}^{n} \times \mathbb{R}^{1}\right.$ ) (this set of functions is dense in $\left.Y_{p}\right)$, we get $\lim _{\varepsilon \rightarrow 0}^{\text {a.e. }}\left(f \otimes K_{\varepsilon}\right)(x, t)=f(x, t)$ for all $f \in Y_{p}$. By the same reason, $\left(f \otimes K_{\varrho}\right)(x, t) \rightarrow 0$ a.e. as $\varrho \rightarrow \infty$ (for nice $f$ this follows from $(3.9$ ) with $p=\infty)$.

The validity of the theorem for weighted wavelet transforms can be proven following the same lines by taking into account that

$$
\int_{\varepsilon}^{\varrho} \mathcal{V}_{\mu} f(x, t ; \eta) \frac{d \eta}{\eta}=f \otimes \widetilde{K}_{\varepsilon}-f \otimes \widetilde{K}_{\varrho}, \quad \widetilde{K}_{\varepsilon}(x, t)=e^{-t} W_{\gamma}(x, t) k_{\varepsilon}(t),
$$

where $k_{\varepsilon}(t)=\varepsilon^{-1} k(t / \varepsilon)$ (similarly for $\widetilde{K}_{\varrho} ;$ cf. (3.6)). In particular,

$$
\begin{aligned}
& \left\|f \otimes \widetilde{K}_{\varepsilon}-c_{\mu} f\right\|_{Y_{p}} \\
& \leq \int_{\mathbb{R}_{+}^{n} \times(0, \infty)} e^{-\varepsilon \tau} W_{\gamma}(y, \tau) k(\tau)\left\|T^{\sqrt{\varepsilon} y, \varepsilon \tau} f(x, t)-f(x, t)\right\|_{Y_{p}} d \nu(y) d \tau \\
& \quad+\|f\|_{Y_{p}} \int_{\mathbb{R}_{+}^{n} \times(0, \infty)}\left|e^{-\varepsilon \tau}-1\right| W_{\gamma}(y, \tau) k(\tau) d \nu(y) d \tau \rightarrow 0 \quad \text { as } \varepsilon \rightarrow 0 .
\end{aligned}
$$

We leave other details to the interested reader.

4. Parabolic potentials associated with the Laplace-Bessel differential operator. The generalized parabolic potentials $H_{\gamma}^{\alpha} f$ and $\mathcal{H}_{\gamma}^{\alpha} f$, defined in terms of the Fourier-Bessel transform by (1.5) and (1.6), can be 
represented as integral operators

$$
\begin{aligned}
& H_{\gamma}^{\alpha} f(x, t)=\frac{1}{\Gamma(\alpha / 2)} \int_{\mathbb{R}_{+}^{n}} \int_{0}^{\infty} \tau^{\alpha / 2-1} W_{\gamma}(y, \tau) T^{y, \tau} f(x, t) d \nu(y) d \tau \\
& \mathcal{H}_{\gamma}^{\alpha} f(x, t)=\frac{1}{\Gamma(\alpha / 2)} \int_{\mathbb{R}_{+}^{n}} \int_{0}^{\infty} \tau^{\alpha / 2-1} e^{-\tau} W_{\gamma}(y, \tau) T^{y, \tau} f(x, t) d \nu(y) d \tau
\end{aligned}
$$

involving the generalized translation (2.4). To see this it suffices to apply the Fourier-Bessel transform (the Fourier transform in $x^{\prime}$ and $t$, and the Bessel transform in $x_{n}$ ) to both sides of (4.1) and (4.2), and make use of the statement (a) of Lemma 2.2. By setting

$$
h_{\alpha}(x, t)=\frac{1}{\Gamma(\alpha / 2)} t_{+}^{\alpha / 2-1} W_{\gamma}(x, t), \quad \widetilde{h}_{\alpha}(x, t)=e^{-t} h_{\alpha}(x, t)
$$

we have $H_{\gamma}^{\alpha} f(x, t)=\left(h_{\alpha} \otimes f\right)(x, t)$ and $\mathcal{H}_{\gamma}^{\alpha} f(x, t)=\left(\widetilde{h}_{\alpha} \otimes f\right)(x, t)$.

The following statement is an analogue of the Hardy-Littlewood-Sobolev theorem (see [6] in the case $\gamma=0$ ).

TheOREM 4.1. (i) Let $1<p<q<\infty, \alpha>0$. The inequality

$$
\left\|H_{\gamma}^{\alpha} f\right\|_{Y_{q}} \leq c\|f\|_{Y_{p}}, \quad c=c(p, q, n, \gamma),
$$

holds for any $f \in Y_{p}$ if and only if $\alpha=(n+2 \gamma-2)\left(p^{-1}-q^{-1}\right)$.

(ii) Define $m\{E\}=\int_{E} d \nu(x) d t$ for $E \subset \mathbb{R}_{+}^{n} \times \mathbb{R}^{1}$. If $f \in Y_{1}$, then

$$
m\left\{(x, t):\left|H_{\gamma}^{\alpha} f(x, t)\right|>\lambda\right\} \leq\left(\frac{c\|f\|_{Y_{1}}}{\lambda}\right)^{q}
$$

for any $\lambda>0$ and $q>1$ such that $1 / q=1-\alpha /(n+2 \gamma-2)$.

(iii) For any $1 \leq p \leq \infty$ and $\alpha>0,\left\|\mathcal{H}_{\gamma}^{\alpha} f\right\|_{Y_{p}} \leq\|f\|_{Y_{p}}$.

Proof. The necessity in (i) is a consequence of the standard homogeneity argument (cf. [18, p. 118]). Further, let

$$
k \equiv k(x, t)=t^{(\alpha-n-2 \gamma) / 2-1} \exp \left(-|x|^{2} /(4 t)\right), \quad x \in \mathbb{R}_{+}^{n}, t>0 .
$$

We split $k$ as $k=k_{1}+k_{2}$, where

$$
k_{1}=\left\{\begin{array}{ll}
k, & t \leq \mu, \\
0, & t>\mu,
\end{array} \quad k_{2}= \begin{cases}0, & t \leq \mu, \\
k, & t>\mu .\end{cases}\right.
$$

Set $m_{0}=m\{k \otimes f>2 \lambda\}$ and $m_{i}=m\left\{k_{i} \otimes f>\lambda\right\}, i=1,2$, and assume $f \geq 0,\|f\|_{Y_{1}}=1$. Then 


$$
\begin{gathered}
m_{0} \leq m_{1}+m_{2}, \quad m_{1} \leq \lambda^{-p}\left\|k_{1} \otimes f\right\|_{Y_{p}}^{p} \stackrel{(2.11)}{\leq} \lambda^{-p}\left\|k_{1}\right\|_{Y_{1}}^{p} \\
\left\|k_{1}\right\|_{Y_{1}}=\int_{0}^{\mu} t^{(\alpha-n-2 \gamma) / 2-1} d t \int_{\mathbb{R}_{+}^{n}} e^{-|x|^{2} /(4 t)} x_{n}^{2 \gamma} d x \\
=c_{n, \gamma} \int_{0}^{\mu} t^{\alpha / 2-1} d t=c_{1} \mu^{\alpha / 2} .
\end{gathered}
$$

Further, by Hölder's inequality,

$$
\begin{aligned}
\left\|k_{2} \otimes f\right\|_{Y_{\infty}} & \leq\left\|k_{2}\right\|_{Y_{p^{\prime}}}=\left(\int_{\mu \mathbb{R}_{+}^{n}}^{\infty}\left[t^{(\alpha-n-2 \gamma) / 2-1} e^{-|x|^{2} /(4 t)}\right]^{p^{\prime}} x_{n}^{2 \gamma} d x\right)^{1 / p^{\prime}} \\
& =c_{p, n, \gamma}\left[\int_{\mu}^{\infty} t^{(\alpha-n-2 \gamma-2) p^{\prime} / 2+(n+2 \gamma) / 2} d t\right]^{1 / p^{\prime}} \\
& =c_{2} \mu^{\beta}, \quad 1 / p^{\prime}+1 / p=1, \\
\beta & =\left(\frac{\alpha-n-2 \gamma-2}{2} p^{\prime}+\frac{n+2 \gamma}{2}+1\right) \frac{1}{p^{\prime}}=-\frac{n+2 \gamma+2}{2 q} .
\end{aligned}
$$

Setting $c_{2} \mu^{\beta}=\lambda$, we have $\left\|k_{2} \otimes f\right\|_{Y_{\infty}} \leq \lambda$ and therefore $m_{2}=0$ (see (4.3)). Hence

$$
m_{0} \stackrel{(4.3)}{\leq} m_{1} \stackrel{(4.4)}{\leq} c \lambda^{-p}\left(\mu^{\alpha / 2}\right)^{p}=c \lambda^{-p} \lambda^{\alpha p / 2 \beta}=c \lambda^{-q}=c\left(\|f\|_{Y_{1}} / \lambda\right)^{q},
$$

and (ii) is proved. By the Marcinkiewicz interpolation theorem this implies the sufficiency in (i). (iii) is a consequence of Young's inequality (2.11) and the fact that $\left\|\widetilde{h}_{\alpha}\right\|_{Y_{1}}=1$.

REmark. For the ordinary Jones-Sampson parabolic potentials, corresponding to $\gamma=0$, an analogue of Theorem 4.1 was stated by V. R. Gopala Rao [6, p. 989]. It is our impression that the proof of the weak $(1, q)$-estimate indicated very briefly in [6] does not yield the required result. In fact, the kernel of the parabolic potential should be split in the $t$-variable (as in our proof) rather than in $x$, as suggested in [6] following Stein's argument [18] applicable to Riesz potentials.

The potentials $H_{\gamma}^{\alpha} f$ and $\mathcal{H}_{\gamma}^{\alpha} f$ can be represented via the relevant wavelet transforms (2.25), (2.26).

LEMMA 4.2. Let $\mu$ be a Borel measure on $[0, \infty)$ such that

$$
\int_{0}^{\infty} \frac{d|\mu|(\tau)}{t^{\alpha / 2}}<\infty \quad \text { and } \quad c_{\alpha, \mu}=\int_{0}^{\infty} \frac{d \mu(\tau)}{\tau^{\alpha / 2}} \neq 0, \quad \alpha>0 .
$$

Then 


$$
\begin{aligned}
& H_{\gamma}^{\alpha} f(x, t)=\frac{c_{\alpha, \mu}^{-1}}{\Gamma(\alpha / 2)} \int_{0}^{\infty} V_{\mu} f(x, t ; \eta) \frac{d \eta}{\eta^{1-\alpha / 2}}, \\
& \mathcal{H}_{\gamma}^{\alpha} f(x, t)=\frac{c_{\alpha, \mu}^{-1}}{\Gamma(\alpha / 2)} \int_{0}^{\infty} \mathcal{V}_{\mu} f(x, t ; \eta) \frac{d \eta}{\eta^{1-\alpha / 2}},
\end{aligned}
$$

provided that the potentials on the left-hand sides are absolutely convergent (i.e. $H_{\gamma}^{\alpha}|f|(x, t)<\infty$ and $\left.\mathcal{H}_{\gamma}^{\alpha}|f|(x, t)<\infty\right)$.

Proof. Owing to (2.25), a simple calculation yields

$$
\begin{aligned}
\int_{0}^{\infty} \frac{V_{\mu} f(x, t ; \eta)}{\eta^{1-\alpha / 2}} d \eta & =\int_{\mathbb{R}_{+}^{n} \times[0, \infty)} W_{\gamma}(y, 1) d \nu(y) d \mu(\tau) \int_{0}^{\infty} T^{\sqrt{\eta \tau} y, \eta \tau} f(x, t) \frac{d \eta}{\eta^{1-\alpha / 2}} \\
& =\int_{\mathbb{R}_{+}^{n} \times(0, \infty)} W_{\gamma}(y, 1) T^{\sqrt{s} y, s} f(x, t) \frac{d \nu(y) d s}{s^{1-\alpha / 2}} \int_{0}^{\infty} \frac{d \mu(\tau)}{\tau^{\alpha / 2}} .
\end{aligned}
$$

By (2.16) this implies (4.5). The proof of (4.6) is similar and based on (2.26).

5. Inversion of generalized parabolic potentials by means of continuous wavelet transforms. By comparing (4.5) and (4.6) with (1.5) and (1.6), it is natural to expect that explicit inverses of $H_{\gamma}^{\alpha}$ and $\mathcal{H}_{\gamma}^{\alpha}$ can be obtained from (4.5) and (4.6) if one replaces (formally) $\alpha$ by $-\alpha$. Indeed, we have the following

Theorem 5.1. Let $\mu$ be a Borel measure supported by $[0, \infty)$ and satisfying the following conditions:

$$
\begin{aligned}
\int_{0}^{\infty} t^{j} d \mu(t)= & 0, \quad j=0,1, \ldots,[\alpha / 2] \text { (the integral part of } \alpha / 2) ; \\
& \int_{0}^{\infty} t^{\beta} d|\mu|(t)<\infty \quad \text { for some } \beta>\alpha / 2 .
\end{aligned}
$$

If $\varphi=H_{\gamma}^{\alpha} f, f \in Y_{p}, 1<p<\infty, 0<\alpha<(n+2 \gamma+2) / p$, then

$$
\int_{0}^{\infty} V_{\mu} \varphi(x, t ; \eta) \frac{d \eta}{\eta^{1+\alpha / 2}}:=\lim _{\varepsilon \rightarrow 0} \int_{\varepsilon}^{\infty}(\ldots)=d_{\alpha, \mu} f(x, t),
$$

where

$$
d_{\alpha, \mu}= \begin{cases}\Gamma(-\alpha / 2) \int_{0}^{\infty} t^{\alpha / 2} d \mu(t) & \text { if } \alpha / 2 \notin \mathbb{N}, \\ \frac{(-1)^{1+\alpha / 2}}{(\alpha / 2) !} \int_{0}^{\infty} t^{\alpha / 2} \log t d \mu(t) & \text { if } \alpha / 2 \in \mathbb{N} .\end{cases}
$$


The limit in (5.3) is interpreted in the $Y_{p}$-norm. The same holds for all $\alpha>0$ and $1 \leq p \leq \infty$ (recall the convention $\left.Y_{\infty} \sim C^{0}\right)$ provided that $H_{\gamma}^{\alpha}$ and $V_{\mu}$ are replaced by $\mathcal{H}_{\gamma}^{\alpha}$ and $\mathcal{V}_{\mu}$, respectively.

Proof. Let us first show that $V_{\mu} H_{\gamma}^{\alpha} f(x, t ; \eta)=\left(f \otimes h_{\eta}^{\alpha / 2}\right)(x, t)$, where

$$
h_{\eta}^{\alpha / 2}(x, t)=\eta^{\alpha / 2-1} W_{\gamma}(x, t)\left(I_{0^{+}}^{\alpha / 2} \mu\right)(t / \eta)
$$

and

$$
\left(I_{0^{+}}^{\alpha / 2} \mu\right)(t)=\frac{1}{\Gamma(\alpha / 2)} \int_{0}^{t}(t-\tau)^{\alpha / 2-1} d \mu(\tau)
$$

is the Riemann-Liouville fractional integral of $\mu$. By (2.25) and (4.1), owing to the properties (2.5)-(2.7) of the generalized translation in the $\xi_{n}$-variable, we have

$$
\begin{aligned}
V_{\mu} \varphi(x, t ; \eta)= & \frac{1}{\Gamma(\alpha / 2)} \int_{0}^{\infty} d \mu(\tau) \int_{\mathbb{R}_{+}^{n}} W_{\gamma}(y, 1) d \nu(y) \int_{0}^{\infty} s^{\alpha / 2-1} d s \\
& \times \int_{\mathbb{R}_{+}^{n}} W_{\gamma}(\xi, s) T_{x, t}^{\sqrt{\eta \tau} y, \eta \tau} T^{\xi, s} f(x, t) d \nu(\xi) \\
= & \frac{1}{\Gamma(\alpha / 2)} \int_{0}^{\infty} d \mu(\tau) \int_{\mathbb{R}_{+}^{n}} W_{\gamma}(y, 1) d \nu(y) \int_{\eta \tau}^{\infty}(\zeta-\eta \tau)^{\alpha / 2-1} d \zeta \\
& \times \int_{\mathbb{R}_{+}^{n}}\left[T^{\sqrt{\eta \tau} y, \eta \tau} W_{\gamma}(\xi, \zeta)\right] T^{\xi, \zeta} f(x, t) d \nu(\xi) \\
= & \frac{1}{\Gamma(\alpha / 2)} \int_{\mathbb{R}_{+}^{n} \times(0, \infty)} T^{\xi, \zeta} f(x, t) d \nu(\xi) d \zeta \\
& \times \int_{0}^{\infty}(\zeta-\eta \tau)_{+}^{\alpha / 2-1} I(\xi, \zeta, \tau) d \mu(\tau),
\end{aligned}
$$

where

$$
I(\xi, \zeta, \tau)=\int_{\mathbb{R}_{+}^{n}} W_{\gamma}(y, 1) T^{\sqrt{\eta \tau} y, \eta \tau} W_{\gamma}(\xi, \zeta) d \nu(y) .
$$

By setting $y=z(\eta \tau)^{-1 / 2}$ and $d \nu(y)=(\eta \tau)^{-\gamma-n / 2} d \nu(z)$, and taking into account that $(\eta \tau)^{-\gamma-n / 2} W_{\gamma}(z / \sqrt{\eta \tau}, 1)=W_{\gamma}(z, \eta \tau)$ (see (2.16)), according to (2.18) we have

$$
I(\xi, \zeta, \tau)=\int_{\mathbb{R}_{+}^{n}} W_{\gamma}(z, \eta \tau) T^{z, \eta \tau} W_{\gamma}(\xi, \zeta) d \nu(z)=W_{\gamma}(\xi, \zeta) .
$$


Hence

$$
\begin{aligned}
V_{\mu} \varphi(x, t ; \eta)= & \frac{1}{\Gamma(\alpha / 2)} \int_{\mathbb{R}_{n}^{+} \times(0, \infty)} W_{\gamma}(\xi, \zeta) T^{\xi, \zeta} f(x, t) \\
& \times\left(\int_{0}^{\infty}(\zeta-\eta \tau)_{+}^{\alpha / 2-1} d \mu(\tau)\right) d \nu(\xi) d \zeta,
\end{aligned}
$$

which gives (5.5). Further,

$$
\int_{\varepsilon}^{\infty} V_{\mu} \varphi(x, t ; \eta) \frac{d \eta}{\eta^{1+\alpha / 2}}=\int_{\mathbb{R}_{+}^{n} \times(0, \infty)} \Psi_{\varepsilon}(\xi, \zeta) T^{\xi, \zeta} f(x, t) d \nu(\xi) d \zeta,
$$

where

$$
\Psi_{\varepsilon}(\xi, \zeta)=\frac{1}{\Gamma(\alpha / 2)} W_{\gamma}(\xi, \zeta) \int_{\varepsilon}^{\infty} \frac{d \eta}{\eta^{1+\alpha / 2}} \int_{0}^{\infty}(\zeta-\eta \tau)_{+}^{\alpha / 2-1} d \mu(\tau) .
$$

A simple calculation yields

$$
\Psi_{\varepsilon}(\xi, \zeta)=\frac{1}{\varepsilon} \lambda_{\alpha}\left(\frac{\zeta}{\varepsilon}\right) W_{\gamma}(\xi, \zeta), \quad \lambda_{\alpha}(t)=\frac{1}{t}\left(I_{0+}^{\alpha / 2+1} \mu\right)(t)
$$

(cf. (3.7), (3.8)). Owing to (5.1) and (5.2), $\lambda_{\alpha}(t)$ has a decreasing integrable majorant and $\int_{0}^{\infty} \lambda_{\alpha}(t) d t=d_{\alpha, \mu}$, with $d_{\alpha, \mu}$ defined by (5.4) (see [15], Lemma 1.3). Hence (5.3) follows by the same arguments as in the proof of Theorem 3.1.

By analogy to (5.6), it can be shown that for $\varphi=\mathcal{H}_{\gamma}^{\alpha} f$,

$$
\int_{\varepsilon}^{\infty} \mathcal{V}_{\mu} \varphi(x, t ; \eta) \frac{d \eta}{\eta^{1+\alpha / 2}}=\left(\widetilde{\Psi}_{\varepsilon} \otimes f\right)(x, t),
$$

where $\widetilde{\Psi}_{\varepsilon}(x, t)=e^{-t} W_{\gamma}(x, t)(1 / \varepsilon) \lambda_{\alpha}(t / \varepsilon)$ and $\mathcal{V}_{\mu} \varphi(x, t ; \eta)$ is defined by (2.26). The convergence $\widetilde{\Psi}_{\varepsilon} \otimes f \rightarrow d_{\alpha, \mu} f$ as $\varepsilon \rightarrow 0$ can be proven as the similar one in Theorem 3.1.

EXAMPLE 5.2. Take a measure $\mu=\sum_{k=0}^{l}(-1)^{k}\left(\begin{array}{l}l \\ k\end{array}\right) \delta_{k}$, where $l>\alpha / 2$ and $\delta_{k}=\delta_{k}(t)$ is the unit mass at $t=k(k=0,1, \ldots, l)$. By $(2.25)$,

$$
\begin{aligned}
V_{\mu} \varphi(x, t ; \eta) & =\int_{\mathbb{R}_{+}^{n}}\left[\sum_{k=0}^{l}(-1)^{k}\left(\begin{array}{l}
l \\
k
\end{array}\right) T^{\sqrt{\eta k} y, \eta k} \varphi(x, t)\right] W_{\gamma}(y, 1) d \nu(y) \\
& =\int_{\mathbb{R}_{+}^{n}} \Delta_{z, \eta}^{l} \varphi(x, t) W_{\gamma}(z, \eta) d \nu(z),
\end{aligned}
$$


where

$$
\Delta_{z, \eta}^{l} \varphi(x, t)=\sum_{k=0}^{l}(-1)^{k}\left(\begin{array}{l}
l \\
k
\end{array}\right) T^{\sqrt{k} z, k \eta} \varphi(x, t)
$$

is an anisotropic finite difference of $\varphi$ generated by the generalized translation operator. Furthermore,

$$
\int_{0}^{\infty} t^{j} d \mu(t)=\sum_{k=0}^{l}(-1)^{k}\left(\begin{array}{l}
l \\
k
\end{array}\right) k^{j}=0, \quad \forall j=0,1, \ldots, l-1
$$

(see [16], pp. 116-117), and (4.2) holds automatically. Thus Theorem 5.1 gives the inversion formula

$$
\int_{\mathbb{R}_{+}^{n} \times(0, \infty)} \frac{\Delta_{z, \eta}^{l} H_{\gamma}^{\alpha} f(x, t)}{\eta^{1+\alpha / 2}} W_{\gamma}(z, \eta) z_{n}^{2 \gamma} d z d \eta=d_{\alpha, \mu} f(x, t)
$$

with

$$
d_{\alpha, \mu}=\int_{0}^{\infty}\left(1-e^{-t}\right)^{l} \frac{d t}{t^{1+\alpha / 2}} \neq 0 .
$$

By the same argument one can obtain an inversion formula for $\mathcal{H}_{\gamma}^{\alpha} f$ :

$$
\begin{gathered}
\int_{\mathbb{R}_{+}^{n} \times(0, \infty)} \frac{\widetilde{\Delta}_{z, \eta}^{l} \mathcal{H}_{\gamma}^{\alpha} f(x, t)}{\eta^{1+\alpha / 2}} W_{\gamma}(z, \eta) z_{n}^{2 \gamma} d z d \eta=d_{\alpha, \mu} f(x, t), \\
\widetilde{\Delta}_{z, \eta}^{l} \varphi(x, t)=\sum_{k=0}^{l}(-1)^{k}\left(\begin{array}{l}
l \\
k
\end{array}\right) e^{-k t} T^{\sqrt{k} z, k \eta} \varphi(x, t) .
\end{gathered}
$$

These formulas were obtained in [12] for potentials with ordinary (euclidean) translation and in $[1,2]$ for the generalized potentials $H_{\gamma}^{\alpha} f$ and $\mathcal{H}_{\gamma}^{\alpha} f$.

\section{References}

[1] I. A. Aliev, On parabolic potentials generated by a generalized shift operator, in: Proc. 3rd Turkish-Azerbaijan Math. Symp., Trabzon, 1993, 14-16.

[2] - The properties and inversion of B-parabolic potentials, in: Special Problems of Math. and Mech., "Bilik", Baku, 1992, 56-75 (in Russian).

[3] I. A. Aliev and S. Bayrakci, On inversion of B-elliptic potentials by the method of Balakrishnan-Rubin, Fract. Calc. Appl. Anal. 1 (1998), 365-384.

[4] I. A. Aliev and B. Rubin, Parabolic wavelet transforms and Lebesgue spaces of parabolic potentials, preprint, The Hebrew University of Jerusalem, 1999.

[5] O. Arena, On a singular parabolic equation related to axially symmetric heat potentials, Ann. Mat. Pura Appl. 105 (1975), 347-393.

[6] V. R. Gopala Rao, A characterization of parabolic function spaces, Amer. J. Math. 99 (1977), 985-993. 
[7] B. F. Jones, Lipschitz spaces and heat equation, J. Math. Mech. 18 (1968), 379-409.

[8] I. A. Kipriyanov, Singular Elliptic Boundary Value Problems, Nauka, Fizmatlit, Moscow, 1997 (in Russian).

[9] B. M. Levitan, Bessel function expansions in series and Fourier integrals, Uspekhi Mat. Nauk 6 (1951), no. 2, 102-143 (in Russian).

[10] J. Löfström and J. Peetre, Approximation theorems connected with generalized translations, Math. Ann. 181 (1969), 255-268.

[11] M. A. Mourou and K. Trimèche, Calderón's reproducing formula associated with the Bessel operator, J. Math. Anal. Appl. 219 (1998), 97-109.

[12] V. A. Nogin and B. S. Rubin, Inversion and description of parabolic potentials with $L_{p}$-densities, Dokl. Akad. Nauk SSSR 284 (1985), 535-538 (in Russian).

[13] B. Rubin, Fractional Integrals and Potentials, Addison-Wesley and Longman, 1996.

[14] - Calderón-type reproducing formula, in: Encyclopaedia Math. Supplement II, Kluwer, 2000, 104-105; reprinted in Frac. Calc. Appl. Anal. 3 (2000), 103-106.

[15] - Fractional integrals and wavelet transforms related to the Blaschke-Levy representation and the spherical Radon transform, Israel J. Math. 114 (1999), 1-27.

[16] S. G. Samko, A. A. Kilbas and O. I. Marichev, Fractional Integrals and Derivatives: Theory and Applications, Gordon and Breach, New York, 1993.

[17] C. H. Sampson, A characterization of parabolic Lebesgue spaces, Dissertation, Rice Univ., 1968.

[18] E. M. Stein, Singular Integrals and Differentiability Properties of Functions, Princeton Univ. Press, Princeton, NJ, 1970.

[19] E. M. Stein and G. Weiss, Introduction to Fourier Analysis on Euclidean Spaces, Princeton Univ. Press, Princeton, NJ, 1971.

[20] K. Stempak, The Littlewood-Paley theory for the Fourier-Bessel transform, preprint no. 45, Univ. of Wrocław, 1985.

[21] —, La théorie de Littlewood-Paley pour la transformation de Fourier-Bessel, C. R. Acad. Sci. Paris Sér. I 303 (1986), 15-18.

[22] S. A. Tersenov, Parabolic Equations with Changing Time Direction, Nauka, Sib. Otdel., Novosibirsk, 1985 (in Russian).

[23] K. Trimèche, Generalized Wavelets and Hypergroups, Gordon and Breach, New York, 1997.

[24] A. Weinstein, Generalized axially symmetric potential theory, Bull. Amer. Math. Soc. 59 (1953), 20-38.

[25] - Singular partial differential equations and their applications, in: Fluid Dynamics and Applied Mathematics, J. B. Diaz and S. I. Pai (eds.), Gordon and Breach, New York, 1962, 229-249.

[26] P. Wojtaszczyk, A Mathematical Introduction to Wavelets, Cambridge Univ. Press, 1997.

Department of Mathematics

Akdeniz University

07058 Antalya, Turkey

E-mail: ialiev@pascal.sci.akdeniz.edu.tr

Institute of Mathematics

The Hebrew University 91904 Jerusalem, Israel E-mail: boris@math.huji.ac.il

Received May 16, 2000

Revised version November 3, 2000 